\title{
Nonlocal surface plasmon amplification by stimulated emission of radiation
}

\author{
Y. Huang, ${ }^{1}$ X. Bian, ${ }^{1}$ Y. X. Ni, ${ }^{1}$ A. E. Miroshnichenko, ${ }^{2}$ and L. Gao ${ }^{1, *}$ \\ ${ }^{1}$ Department of Physics, Soochow University, 1 Shizi Street, Suzhou 215006, China \\ ${ }^{2}$ Nonlinear Physics Centre, Australian National University, Canberra, Australian Capital Territory 0200, Australia
}

(Received 23 February 2014; published 15 May 2014)

\begin{abstract}
We establish the spaser generation conditions for nonlocal plasmonic lasers and nonlocal plasmonic core-shell lasers based on the full-wave nonlocal Mie theory. Numerical results show that the required gain threshold and the gain refractive index become large generally when the nonlocality or spatial dispersion is taken into account. This tendency can be understood by the analysis with the proposed equivalent permittivity for nonlocal metallic nanoparticles. Since the nonlocality for the compact nanoparticles is quite significant, our study will be of great importance in the design of ultrasmall nanoparticle lasers.
\end{abstract}

DOI: 10.1103/PhysRevA.89.053824

PACS number(s): 42.55.-f, 42.25.Fx, 73.20.Mf, 78.67.Bf

\section{INTRODUCTION}

Nanoparticle lasers (NPLs) attract more and more attention due to their potential applications in biosensing, data storage, optical communications, medical applications, and so on [1-4]. Based on the strong interactions between nanosized emitters and localized surface plasmons (SPs), this kind of lasers is also called "spaser" (short for surface plasmon amplification by stimulated emission of radiation), which can generate the coherent plasmonic fields in the subwavelength regions [5]. In comparison with the conventional lasers built on the dielectric cavities, the spaser can beat the diffraction limit and focus electromagnetic waves to spots much smaller than the wavelength. For spasers, the SP modes can provide feedback on the nanometer scale and can be amplified as long as the dissipative losses are compensated by the gain medium around metal nanostructures. Recently, the original theoretical proposal of the spaser was demonstrated experimentally [6] with nanoparticles containing a gold core and dye-doped silica shell, from which it can be understood that the core-shell nanoparticle itself serves as a resonator (or a resonant cavity) and the adjacent gain medium delivers energy to the SP mode.

However, due to strong metallic losses particularly at optical frequencies, the required gain threshold to achieve lasing from such a nanosize cavity is extremely high. Actually, a spaser with a large and/or unattainable lasing threshold due to high dissipative loss is undesirable for practical applications. In this connection, a lot of research efforts were made to decrease the lasing threshold. For instance, $\mathrm{Li}$ and $\mathrm{Yu}$ [7] achieved low-threshold gain by embedding the Au-nanoparticle lasers into the medium with high refractive index. Pan et al. [8] introduced low-threshold plasmonic lasing based on the high- $Q$ dipole void mode in a metallic nanoshell. Calander et al. [9] studied the characteristics of the plasmonic core-shell nanoparticle lasers and found that the laser made of $\mathrm{Ag}$ material possesses lower gain threshold than that of Au. Moreover, the nanorod spaser [10,11], symmetry-broken plasmonic core-shell spaser [12], magneto-optical spaser [13], and electric spaser [14] were also studied.

On the other hand, SP modes on metal nanostructures can confine the electromagnetic energy to very small volume,

\footnotetext{
*leigao@suda.edu.cn
}

and the dimension of NPLs can be at the tens-of-nanometers level [5-7]. In this regard, nonlocal theory should be adopted because of its very accurate description of electromagnetic (EM) properties in comparison with local theory $[15,16]$. The nonlocality arises from the electron-electron interactions in the dielectric response of metals [17] and leads to the spatial dispersion of metals. As a consequence, it shall play a further role in the metal's SPs. Actually, the nonlocality (or spatial dispersion) was found to diminish the impact caused by geometric imperfections [18], reduce the field enhancement of the nanostructures, and make the plasmon resonant frequency blueshift [19-26]. For NPLs composed of metal with very small sizes, their lasing properties should be affected by the nonlocality. To the best of our knowledge, these nonlocal effects on the NPLs have not been theoretically explored so far. In this paper, we shall employ full-wave nonlocal Mie scattering theory to obtain the spaser generation conditions for a nonlocal gain-assistant nanosphere and coreshell nanosphere lasers. Moreover, we pay much attention to the choices of physical parameters in the design of spasers with plasmonic nanostructures adjacent to the gain medium when we take into account the spatial dispersion. It is demonstrated that light scattering provides a simple diagnostics method to assess how far a specific nanoparticle is from reaching the lasing threshold [9], and the singularities in scattering parameters can be used to derive the lasing threshold for the spaser $[4,7,13]$. This problem is similar to the external wave driven laser generation too [27].

The paper is organized as follows. In Sec. II, we employ full-wave nonlocal Mie scattering theory to obtain the spaser generation conditions for nonlocal gain-assistant nanosphere and metal core-gain shell nanosphere lasers, respectively. In Sec. III, numerical results about nonlocal effects on the threshold gain are shown. Our conclusions and discussions are presented in Sec. IV.

\section{MODEL AND THEORY}

First, let us consider the spaser consisting of a nonlocal metallic $\mathrm{Au}$ sphere with the radius $r_{\mathrm{c}}$ embedded in a semiinfinite host gain medium with permittivity $\varepsilon_{\mathrm{G}}$. Without loss of generality, we assume that the metal is described with the Drude permittivity $\varepsilon_{\mathrm{T}}$ for the transverse electric fields and a spatially dispersive permittivity $\varepsilon_{\mathrm{L}}$ for the longitudinal electric 
fields, which are written as [28,29]

$$
\varepsilon_{\mathrm{T}}(\omega)=\varepsilon_{\mathrm{g}}+\varepsilon_{\mathrm{h}}(\omega, 0), \quad \varepsilon_{\mathrm{L}}(\omega)=\varepsilon_{\mathrm{g}}+\varepsilon_{\mathrm{h}}(\omega, k),
$$

where $\varepsilon_{\mathrm{g}}$ is the background permittivity of the metal related to the interband transitions, and the hydrodynamic part $\varepsilon_{\mathrm{h}}(\omega, k)$ is given by

$$
\varepsilon_{\mathrm{h}}(\omega, k)=\frac{-\omega_{\mathrm{p}}^{2}}{\omega(\omega+i \gamma)-\beta^{2} k^{2}},
$$

where $\omega_{\mathrm{p}}$ and $\gamma$ are the plasma frequency and the damping constant. By taking into account the size-dependent electron scattering, one yields $\gamma=\gamma_{\infty}+A v_{\mathrm{F}} / r_{\mathrm{c}}\left(v_{\mathrm{F}}\right.$ is the Fermi velocity and $A$ is the fitting constant factor determined by the geometry and theory) [30]. In addition, $\beta$ measures the degree of nonlocality, which is proportional to the Fermi velocity $v_{\mathrm{F}}$. The wave vector of the longitudinal plasmon wave is determined by the equation $\varepsilon_{\mathrm{L}}\left(\omega, k_{\mathrm{L}}\right)=0$, while the transverse electromagnetic mode satisfies the conventional dispersion law $k_{\mathrm{T}}^{2}=(\omega / c)^{2} \varepsilon_{\mathrm{T}}(\omega)$.

The incident electric field can be expanded as

$$
\begin{aligned}
E^{\mathrm{I}}= & E_{0} e^{-i \omega t} \sum_{n=1}^{\infty} i^{n} \frac{2 n+1}{n(n+1)} \\
& \times\left\{\nabla \times\left[\vec{r} \cdot j_{n}\left(k_{\mathrm{G}} r\right) P_{n}^{(1)}(\cos \theta) \sin \phi\right]\right. \\
& \left.-i \frac{1}{k_{\mathrm{G}}} \nabla \times \nabla \times\left[\vec{r} \cdot j_{n}\left(k_{\mathrm{G}} r\right) P_{n}^{(1)}(\cos \theta) \cos \phi\right]\right\},
\end{aligned}
$$

and the scattering wave is written as

$$
\begin{aligned}
E^{\mathrm{R}}= & E_{0} e^{-i \omega t} \sum_{n=1}^{\infty} i^{n} \frac{2 n+1}{n(n+1)} \\
& \times\left\{\nabla \times\left[\vec{r} \cdot b_{n} h_{n}\left(k_{\mathrm{G}} r\right) P_{n}^{(1)}(\cos \theta) \sin \phi\right]\right. \\
& \left.-i \frac{1}{k_{\mathrm{G}}} \nabla \times \nabla \times\left[\vec{r} \cdot a_{n} h_{n}\left(k_{\mathrm{G}} r\right) P_{n}^{(1)}(\cos \theta) \cos \phi\right]\right\},
\end{aligned}
$$

where $j_{n}(x)$ [or $h_{n}(x)$ ] is the spherical Bessel (or Hankel) function of the first kind, $P_{n}^{(1)}(\cos \theta)$ is the associated Legendre polynomial, and $k_{\mathrm{G}}=(\omega / c)\left(\varepsilon_{\mathrm{G}}\right)^{1 / 2}$ is the wave number of transverse waves in the gain medium.

The electric fields associated with the transverse and longitudinal waves excited in the nonlocal $\mathrm{Au}$ nanoparticle are written as

$$
\begin{gathered}
E^{\mathrm{T}}=E_{0} e^{-i \omega t} \sum_{n=1}^{\infty} i^{l} \frac{2 n+1}{n(n+1)} \\
\times\left\{\nabla \times\left[\vec{r} \cdot c_{n} j_{n}\left(k_{\mathrm{T}} r\right) P_{n}^{(1)}(\cos \theta) \sin \phi\right]\right. \\
\left.-i \frac{1}{k_{\mathrm{T}}} \nabla \times \nabla \times\left[\vec{r} \cdot d_{n} j_{n}\left(k_{\mathrm{T}} r\right) P_{n}^{(1)}(\cos \theta) \cos \phi\right]\right\}, \\
E^{\mathrm{L}}=E_{0} e^{-i \omega t} \sum_{n=1}^{\infty} i^{n} \frac{2 n+1}{n(n+1)} \\
\cdot \nabla\left[g_{n} j_{n}\left(k_{\mathrm{L}} r\right) P_{n}^{(1)}(\cos \theta) \cos \phi\right] .
\end{gathered}
$$

We remind the reader that there is no magnetic field for the longitudinal mode.

Five unknown coefficients $\left(a_{n}, b_{n}, c_{n}, d_{n}\right.$, and $\left.g_{n}\right)$ could be determined by imposing the boundary conditions on the interface at $r=r_{\mathrm{c}}$. In general, the continuity conditions of the tangential components of the electric and magnetic fields would be matched on the interface. Besides that, because of the excitation of the additional longitudinal mode inside the metallic nanoparticle, an additional boundary condition is required. Here, we choose the vanishing normal component of the exciton polarization vector at the surface as the additional boundary condition [31]. Based on full-wave nonlocal Mie theory [15,31], we can derive the scattering coefficients $a_{n}$ and $b_{n}$, which are related to electric and magnetic scattering channels. For the nonmagnetic system, $a_{n}$ dominates the polarizability of the nanoparticle. In this case, we have

$$
a_{n}=\frac{N_{n}}{D_{n}},
$$

where

$$
D_{n}=\left|\begin{array}{ccc}
-\frac{\left[k_{\mathrm{G}} r_{\mathrm{c}} h_{n}\left(k_{\mathrm{G}} r_{\mathrm{c}}\right)\right]^{\prime}}{k_{\mathrm{G}} r_{\mathrm{c}}} & \frac{\left[k_{\mathrm{T}} r_{\mathrm{c}} j_{n}\left(k_{\mathrm{T}} r_{\mathrm{c}}\right)\right]^{\prime}}{k_{\mathrm{T}} r_{\mathrm{c}}} & -\frac{j_{n}\left(k_{\mathrm{L}} r_{\mathrm{c}}\right)}{k_{\mathrm{L}} r_{\mathrm{c}}} \\
-\sqrt{\varepsilon_{\mathrm{G}}} h_{n}\left(k_{\mathrm{G}} r_{\mathrm{c}}\right) & \sqrt{\varepsilon_{\mathrm{T}}} j_{n}\left(k_{\mathrm{T}} r_{\mathrm{c}}\right) & 0 \\
0 & V_{n} & -\varepsilon_{\mathrm{g}} j_{n}^{\prime}\left(k_{\mathrm{L}} r_{\mathrm{c}}\right)
\end{array}\right|,
$$

and $N_{n}$ differs from that of $D_{n}$ only by the first row in which the symbols $h_{n}$ are replaced by $j_{n}$, while the minus sign is removed. $j_{n}^{\prime}(x)$ [or $h_{n}^{\prime}(x)$ ] denotes $\partial j_{n}(x) / \partial x$ [or $\partial h_{n}(x) / \partial x$ ] and $V_{n}=n(n+1)\left(\varepsilon_{\mathrm{g}}-\varepsilon_{\mathrm{T}}\right) j_{n}\left(k_{\mathrm{T}} r_{\mathrm{c}}\right) /\left(k_{\mathrm{T}} r_{\mathrm{c}}\right)$.

For the dipole nanoparticle, the total scattering property is dominated by the $n=1$ term. Thus, the condition for the spaser generation condition is expressed as

$$
\begin{aligned}
D_{1}= & \frac{\left[k_{\mathrm{G}} r_{\mathrm{c}} h_{1}\left(k_{\mathrm{G}} r_{\mathrm{c}}\right)\right]^{\prime}}{k_{\mathrm{G}} r_{\mathrm{c}}} \sqrt{\varepsilon_{\mathrm{T}}} j_{1}\left(k_{\mathrm{T}} r_{\mathrm{c}}\right) \varepsilon_{\mathrm{g}} j_{1}^{\prime}\left(k_{\mathrm{L}} r_{\mathrm{c}}\right) \\
& +\frac{j_{1}\left(k_{\mathrm{L}} r_{\mathrm{c}}\right)}{k_{\mathrm{L}} r_{\mathrm{c}}} \sqrt{\varepsilon_{\mathrm{G}}} h_{1}\left(k_{\mathrm{G}} r_{\mathrm{c}}\right) V_{1} \\
& -\frac{\left[k_{\mathrm{T}} r_{\mathrm{c}} j_{1}\left(k_{\mathrm{T}} r_{\mathrm{c}}\right)\right]^{\prime}}{k_{\mathrm{T}} r_{\mathrm{c}}} \sqrt{\varepsilon_{\mathrm{G}}} h_{1}\left(k_{\mathrm{G}} r_{\mathrm{c}}\right) \varepsilon_{\mathrm{g}} j_{1}^{\prime}\left(k_{\mathrm{L}} r_{\mathrm{c}}\right)=0 .
\end{aligned}
$$

Equation (9) is nothing but the singularity condition for the nonlocal sphere [32]. In the following section, this transcendental equation would be numerically solved in order to get the required refractive index of the gain medium $n_{\mathrm{G}}=\sqrt{\varepsilon_{\mathrm{G}}}$ to achieve singularity of the field intensity, and the threshold gain $G_{\text {th }}=-4 \pi \operatorname{Im}\left(n_{\mathrm{G}}\right) / \lambda$ can be obtained as well. Within the electrostatic approximation, we have $x=k_{\mathrm{G}} r_{\mathrm{c}}$ (or $\left.k_{\mathrm{T}} r_{\mathrm{c}}\right) \ll 1$. Expanding $j_{1}(x)$ [or $j_{1}^{\prime}(x)$ ], $h_{1}(x)$ [or $h_{1}^{\prime}(x)$ ] into a Taylor series yields

$$
3\left[\varepsilon_{\mathrm{T}}+2 \varepsilon_{\mathrm{G}}-2 \varepsilon_{\mathrm{G}} Q\right]-i 2 k_{\mathrm{G}}^{3} r_{\mathrm{c}}^{3}\left[\varepsilon_{\mathrm{T}}-\varepsilon_{\mathrm{G}}+\varepsilon_{\mathrm{G}} Q\right]=0 .
$$

By neglecting the high-order term of $k_{\mathrm{G}} r_{\mathrm{c}}$, the spaser generation condition in the electrostatic approximation is

$$
\varepsilon_{\mathrm{T}}+2 \varepsilon_{\mathrm{G}}-2 \varepsilon_{\mathrm{G}} Q=0,
$$

with $Q=\left(\varepsilon_{\mathrm{g}}-\varepsilon_{\mathrm{T}}\right) j_{1}\left(k_{\mathrm{L}} r_{\mathrm{c}}\right) /\left[\varepsilon_{\mathrm{g}} k_{\mathrm{L}} r_{\mathrm{c}} j_{1}^{\prime}\left(k_{\mathrm{L}} r_{\mathrm{c}}\right)\right]$. Note that Eq. (11) is just the surface-plasmon resonant condition for the nonlocal sphere in the electrostatic approximation. If the spatial dispersion of the metal is not taken into account, the 
longitudinal mode cannot be supported in the nanoparticle any more, and the imaginary part of $k_{\mathrm{L}}$ will become infinitely large [15]. Substituting $\operatorname{Im}\left(k_{\mathrm{L}}\right) \rightarrow \infty$ into Eq. (11) results in the resonant condition within local descriptions $\varepsilon_{\mathrm{T}}+2 \varepsilon_{\mathrm{G}}=$ $0[33,34]$. Therefore, the required refractive index of the gain medium to achieve the singularity condition has the form

$$
n_{\mathrm{G}}=\sqrt{-\frac{\varepsilon_{\mathrm{T}}}{2(1-Q)}} .
$$

In reality, the gain medium cannot be infinitely extended to the whole space, and we would like to study the Au core-gain shell nanoparticle laser $[6,7,9]$. In this situation, the scattering coefficient $a_{n}$ for a nonlocal core-dielectric shell nanoparticle can be similarly derived by imposing two conventional EM boundary conditions in the two interfaces ( $r=r_{\mathrm{s}}$ and $r=r_{\mathrm{c}}$ ) and an additional one in the inner interface $\left(r=r_{\mathrm{c}}\right)$ [26]. After some tedious derivations, we obtain the scattering coefficient

$$
a_{n}=\frac{N_{n}}{D_{n}},
$$

with

$$
D_{n}=\left|\begin{array}{ccccc}
-\frac{\left[k_{\mathrm{A}} r_{\mathrm{s}} h_{n}\left(k_{\mathrm{A}} r_{\mathrm{s}}\right)\right]^{\prime}}{k_{\mathrm{A}} r_{\mathrm{s}}} & \frac{\left[k_{\mathrm{G}} r_{\mathrm{s}} j_{n}\left(k_{\mathrm{G}} r_{\mathrm{s}}\right)\right]^{\prime}}{k_{G}} & \frac{\left[k_{\mathrm{G}} r_{\mathrm{s}} \mathrm{y}_{n}\left(k_{\mathrm{G}} r_{\mathrm{s}}\right)\right]^{\prime}}{k_{\mathrm{G}} r_{\mathrm{s}}} & 0 & 0 \\
-\sqrt{\varepsilon_{\mathrm{A}}} h_{n}\left(k_{\mathrm{A}} r_{\mathrm{s}}\right) & \sqrt{\varepsilon_{\mathrm{G}}} j_{n}\left(k_{\mathrm{G}} r_{\mathrm{s}}\right) & \sqrt{\varepsilon_{\mathrm{G}} \mathrm{y}_{n}\left(k_{\mathrm{G}} r_{\mathrm{s}}\right)} & 0 & 0 \\
0 & \frac{\left[k_{\mathrm{G}} r_{\mathrm{c}} j_{n}\left(k_{\mathrm{G}} r_{\mathrm{c}}\right)\right]^{\prime}}{k_{\mathrm{G}} r_{\mathrm{c}}} & \frac{\left[k_{\mathrm{G}} r_{\mathrm{c}} \mathrm{y}_{n}\left(k_{\mathrm{G}} r_{\mathrm{c}}\right)\right]^{\prime}}{k_{\mathrm{G}} r_{\mathrm{c}}} & -\frac{\left[k_{\mathrm{T}} r_{\mathrm{c}} j_{n}\left(k_{\mathrm{T}} r_{\mathrm{c}}\right)\right]^{\prime}}{k_{\mathrm{T}} r_{\mathrm{c}}} & \frac{j_{n}\left(k_{\mathrm{L}} r_{\mathrm{c}}\right)}{k_{\mathrm{L}} r_{\mathrm{c}}} \\
0 & \sqrt{\varepsilon_{\mathrm{G}} j_{n}\left(k_{\mathrm{G}} r_{\mathrm{c}}\right)} & \sqrt{\varepsilon_{\mathrm{G}} \mathrm{y}_{n}\left(k_{\mathrm{G}} r_{\mathrm{c}}\right)} & -\sqrt{\varepsilon_{\mathrm{T}}} j_{n}\left(k_{\mathrm{T}} r_{\mathrm{c}}\right) & 0 \\
0 & 0 & 0 & V_{n} & -\varepsilon_{\mathrm{g}}\left[j_{n}\left(k_{\mathrm{L}} r_{\mathrm{c}}\right)\right]
\end{array}\right| .
$$

Again, $N_{n}$ has a similar form with $D_{n}$ except that the symbols $h_{n}$ in the first row are replaced by $j_{n}$ and the minus sign is removed. Then, we establish the spaser generation condition for nonlocal Au core-gain shell nanospheres under which the singularity occurs, i.e.,

$$
D_{1}=0 \text {. }
$$

Within the electrostatic approximation, Eq. (15) is simplified as

$$
\left(\frac{r_{\mathrm{s}}}{r_{\mathrm{c}}}\right)^{3}=\frac{2\left[(1-Q) \varepsilon_{\mathrm{G}}-\varepsilon_{\mathrm{T}}\right]\left(\varepsilon_{\mathrm{G}}-\varepsilon_{\mathrm{A}}\right)}{\left[\varepsilon_{\mathrm{T}}+2(1-Q) \varepsilon_{\mathrm{G}}\right]\left(\varepsilon_{\mathrm{G}}+2 \varepsilon_{\mathrm{A}}\right)},
$$

where $\varepsilon_{\mathrm{A}}$ is the permittivity of the surrounding medium. Incidentally, if we neglect the nonlocality, i.e., $Q=0$, Eq. (16) is reduced to the local one as expected.

\section{NUMERICAL RESULTS}

We are now in a position to give some numerical results about the nonlocal effects on the nanolasers. For numerical calculations, we choose $\varepsilon_{\mathrm{g}}=\varepsilon_{\infty}+\varepsilon_{x 1}+\varepsilon_{x 2}$ as adopted in Ref. [7], and $\omega_{\mathrm{p}}=1.32 \times 10^{16} \mathrm{~s}^{-1}, \gamma_{\infty}=3.3095 \times 10^{13} \mathrm{~s}^{-1}$, $\beta=\sqrt{3 / 5} v_{\mathrm{F}}, A=1$. It was reported that the electrostatic approximation could be misleading even in nanoscale optics [29]. Here, we define the relative error of the electrostatic approximation as

$$
\begin{aligned}
& E_{\mathrm{Re}}=\left|\frac{\operatorname{Re}\left(n_{\mathrm{G}}\right)-\operatorname{Re}\left(\tilde{n}_{\mathrm{G}}\right)}{\operatorname{Re}\left(n_{\mathrm{G}}\right)}\right| \times 100 \%, \\
& E_{\mathrm{Im}}=\left|\frac{\operatorname{Im}\left(n_{\mathrm{G}}\right)-\operatorname{Im}\left(\tilde{n}_{\mathrm{G}}\right)}{\operatorname{Im}\left(n_{\mathrm{G}}\right)}\right| \times 100 \%,
\end{aligned}
$$

where $n_{\mathrm{G}}$ in Eq. (17) is obtained by solving Eq. (9) numerically and $\tilde{n}_{\mathrm{G}}$ is determined by Eq. (12). The results for the relative error are shown in Fig. 1 for both nonlocal and local cases. For the $\mathrm{Au}$ sphere embedded in the gain medium with radius $r_{\mathrm{c}}=$
$10 \mathrm{~nm}$, the difference for the relative error between nonlocal and local cases is quite small and may become large in the long-wavelength region. Interestingly, the relative errors of the electrostatic approximation are within $10 \%$ in the whole wavelength range. In what follows, $n_{\mathrm{G}}$ is obtained based on Eq. (9) or (15), which is beyond the electrostatic approximation.

To illustrate the nonlocal effects on the gain threshold and the real part of $n_{\mathrm{G}}$, it is helpful to define the parameters $\delta=$ $\left(G_{\text {th-non }}-G_{\text {th }}\right) / G_{\text {th }}$ and $\xi=\left[\operatorname{Re}\left(n_{\text {th-non }}\right)-\operatorname{Re}\left(n_{\mathrm{G}}\right)\right] / \operatorname{Re}\left(n_{\mathrm{G}}\right)$ to make comparisons between the local case $G_{\text {th }}\left[\operatorname{Re}\left(n_{\mathrm{G}}\right)\right]$ and the nonlocal case $G_{\text {th-non }}\left[\operatorname{Re}\left(n_{\mathrm{G}-\mathrm{non}}\right)\right]$ for the gain-assistant single nanoparticle laser.

$\delta$ and $\xi$ are plotted as functions of $r_{\mathrm{c}}$ and the incident wavelength $\lambda$ in Figs. 2(a) and 2(b). Generally, both $G_{\text {th-non }}$ and $\operatorname{Re}\left(n_{\mathrm{G}-\mathrm{non}}\right)$ for the nonlocal case are larger than $G_{\text {th }}$ and $\operatorname{Re}\left(n_{\mathrm{G}}\right)$ for the local case, and $\delta$ and $\xi$ can be larger than $5 \%$ especially for small $r_{\mathrm{c}}$ and long wavelength. For $r_{\mathrm{c}}>3 \mathrm{~nm}$, with the decrease of $r_{\mathrm{c}}$, the nonlocality plays

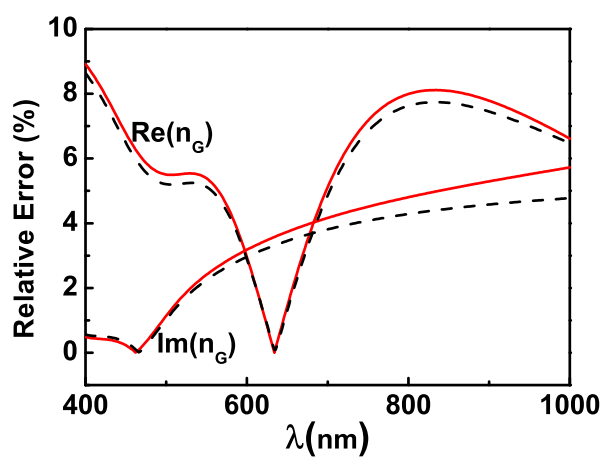

FIG. 1. (Color online) Relative error of the electrostatic approximation found within nonlocal theory (the red solid line) and the local one (the black dashed line). The radius of the nonlocal sphere is $10 \mathrm{~nm}$. 


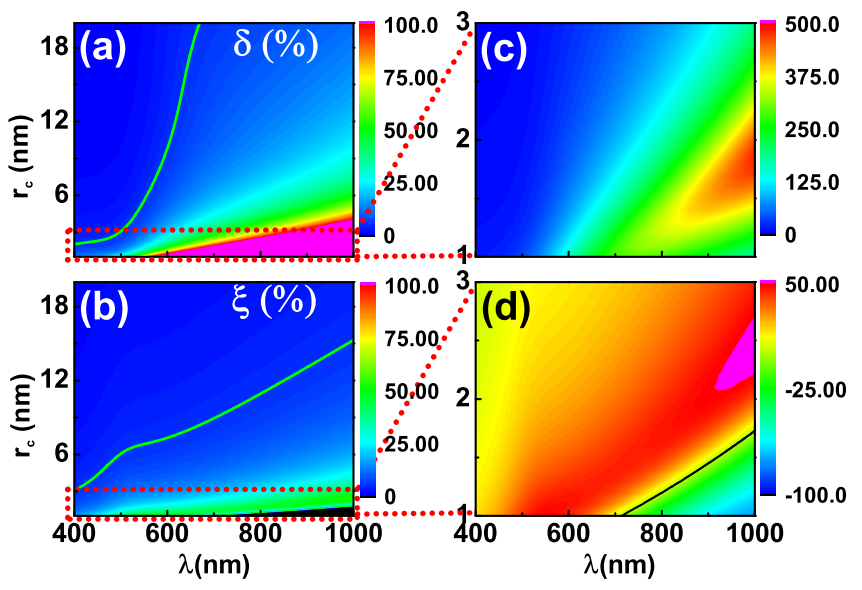

FIG. 2. (Color online) Images of (a) $\delta$ and (b) $\xi$ in the plane of $r_{\mathrm{c}}$ and $\lambda$. (c) and (d) Part-amplified versions of (a) and (b) with different colorbar values, respectively. Green lines (light gray) in (a) and (b) represent the value $5 \%$ and the black line in (d) is the $0 \%$ line.

a more and more important role in the gain threshold and the gain refractive index. The large discrepancy between the nonlocal and local cases for Au nanoparticles with smaller size results mainly from the intrinsic quantum properties of the metal's conduction electrons [17]. These screening electrons are distributed in a layer on the particle's surface and have much effect on the interaction between metal and incident light if this layer thickness is comparable to the size of nanoparticles. Actually, the Au nanoparticle acts as a laser cavity [1], and the introduction of the spatial dispersion (or nonlocality) diminishes the strength of the excited local fields by the SPs. As a consequence, in order to give the same radiation, we need a very large threshold of the surrounding gain when the nonlocality is taken into account. In addition, the longer the incident wavelength, the larger $\delta$ and $\xi$ are. This is due to the fact that the denominator of $\varepsilon_{\mathrm{h}}(\omega, k)$ is dominated by the $\beta^{2} k^{2}$ term for large $\lambda$ (or small $\omega$ ). However, situations are dramatically different for $r_{\mathrm{c}}<3 \mathrm{~nm}$ as shown in Figs. 2(c) and 2 (d). Here $\xi$ is found to be lower than zero (indicated by the black line) in an ultrasmall region [see Fig. 2(d)]. This indicates that one may need a smaller gain refractive index under the nonlocal descriptions than in the local case to realize the lasing state. In addition, $\delta$ may achieve much magnitude and the required gain threshold is extremely large for both $r_{\mathrm{c}}<3 \mathrm{~nm}$ and $\lambda>800 \mathrm{~nm}$, which should be avoided. Therefore, it is very important to consider these deviations, especially the dramatic change in threshold gain $G_{\text {th }}$ and required $\operatorname{Re}\left(n_{\mathrm{G}}\right)$, if one promises the candidates toward ultrasmall nanoparticle lasers.

The above phenomena could also be further understood by our recently proposed equivalent permittivity (EP) for a nonlocal metallic nanoparticle [26], which is written as

$$
\varepsilon_{\text {eq }}=\frac{j_{1}\left(k_{\mathrm{T}} r_{\mathrm{c}}\right)}{k_{\mathrm{T}} r_{\mathrm{c}} j_{1}^{\prime}\left(k_{\mathrm{T}} r_{\mathrm{c}}\right)-Q j_{1}\left(k_{\mathrm{T}} r_{\mathrm{c}}\right)} \varepsilon_{\mathrm{T}} .
$$

In Fig. 3 we show the imaginary and real parts of EP for both nonlocal and local metallic spheres, respectively, as a function of $\lambda$. It is evident that the equivalent damping for the nonlocal nanoparticle is higher than the local one for a definite $\lambda$, and

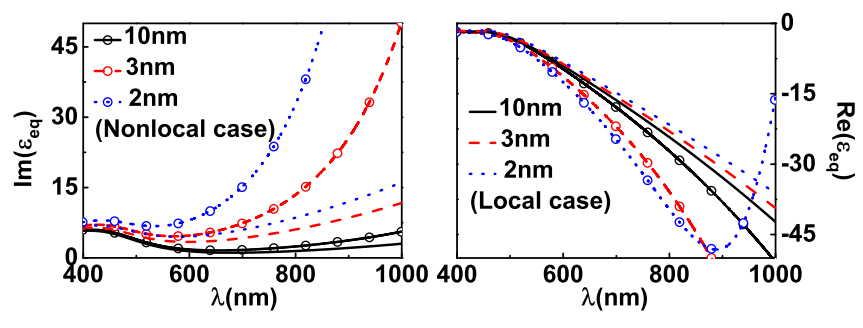

FIG. 3. (Color online) Imaginary and real parts of the nonlocal equivalent permittivity $\varepsilon_{\text {eq }}$ and the local permittivity $\varepsilon_{\mathrm{T}}$ for $\mathrm{Au}$ nanoparticles as functions of $\lambda$ with various radii $r=10,3$, and $2 \mathrm{~nm}$.

hence the gain required is higher as expected. On the other hand, the real part of EP is more negative than the local one, which leads to a larger required refractive index to achieve the resonant state.

Next, we perform the numerical calculations on the coreshell nanoparticle lasers for the outer radius $r_{\mathrm{s}}=20 \mathrm{~nm}$. In Figs. 4(a) and 4(b), the dependence of $G_{\text {th-non }}$ and $\operatorname{Re}\left(n_{\text {G-non }}\right)$ for the nonlocal metal core-gain shell nanosphere on $\lambda$ and the inner radius $r_{\mathrm{c}}$ are shown. It is found that for a given wavelength $(\lambda>600 \mathrm{~nm})$ there is an optimal $r_{\mathrm{c}}$ for one to achieve the minimal gain threshold [see Fig. 4(a)]. In detail, the gain threshold shows a rapid decrease as the inner radius $r_{\mathrm{c}}$ is increased from $3 \mathrm{~nm}$, reaches a minimum, and then increases rapidly with further increasing $r_{\mathrm{c}}$ for $r>16 \mathrm{~nm}$ [see Fig. 4(c)]. This nonmonotonic dependence can be well understood: for a small nonlocal metallic core, the damping is quite high due to the finite-size effect, and hence more gain threshold is required to compensate the metallic losses; on the other hand, since the shell radius is finite, the large size of the metallic core leads to the small volume fraction of the gain shell, and an increased gain threshold value is expected again. Note that we also find a broad region of low gain threshold where $r_{\mathrm{c}}$ is
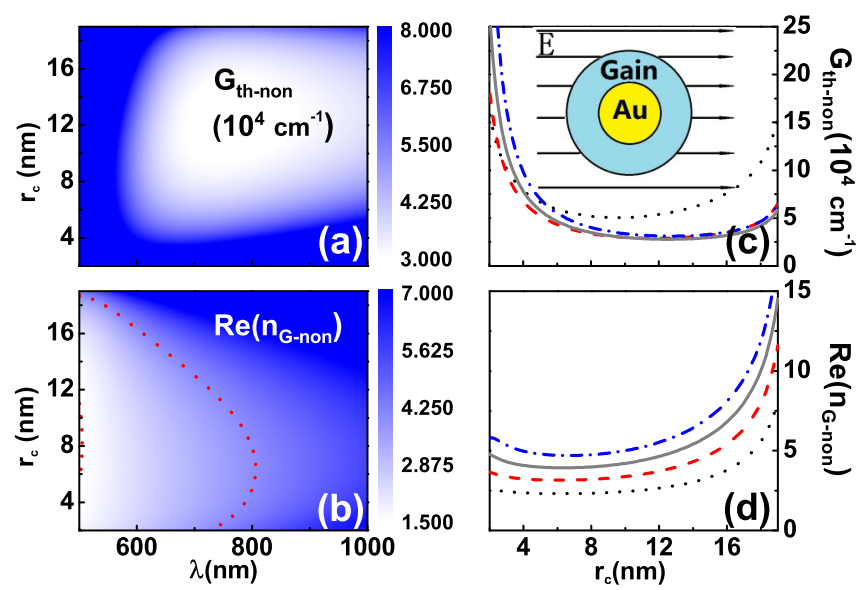

FIG. 4. (Color online) Images of (a) $G_{\text {th-non }}$ and (b) $\operatorname{Re}\left(n_{\mathrm{G}-\text { non }}\right)$ in the plane of $r_{\mathrm{c}}$ and $\lambda$ with $\varepsilon_{\mathrm{A}}=1$. Plots of (c) $G_{\text {th-non }}$ and (d) $\operatorname{Re}\left(n_{\mathrm{G}-\text { non }}\right)$ vs $r_{\mathrm{c}}$ for various $\lambda$, i.e., $600 \mathrm{~nm}$ (the dotted black line), $700 \mathrm{~nm}$ (the dashed red line), $800 \mathrm{~nm}$ (the solid gray line), and $900 \mathrm{~nm}$ (the dash-dotted blue line). The approximate range for a realistic $\operatorname{Re}\left(n_{\mathrm{G}}\right)$ $(1.5-4)$ is marked as red dots in (b). Inset: Schematic model of the nonlocal core-shell particle laser. 


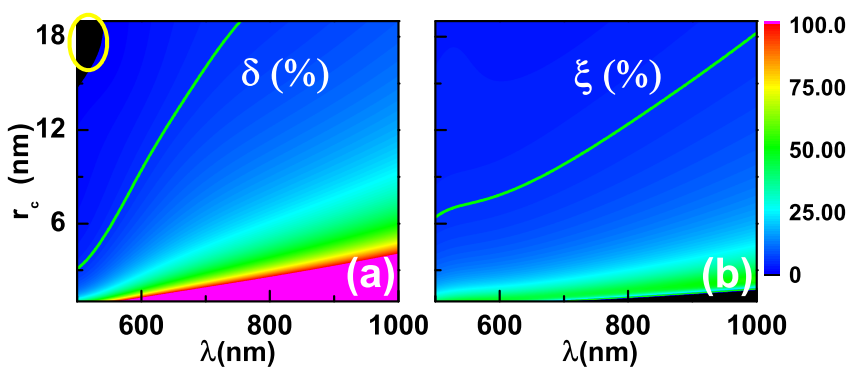

FIG. 5. (Color online) Images of (a) $\delta$ and (b) $\xi$ in the plane of $r_{\mathrm{c}}$ and $\lambda$. Green (light gray) lines in (a) and (b) represent the value $5 \%$.

in the range between 4 and $16 \mathrm{~nm}$ [see Fig. 4(c)]. That is to say, the required threshold is almost unchanged although the volume fraction of the gain shell is decreased. As to $\operatorname{Re}\left(n_{\mathrm{G}-\mathrm{non}}\right)$, similar conclusions are made [see Figs. 4(b) and 4(d)]. In reality, the quantum dots and active molecules (fluorescence or dye molecules) may be the typical gain medium materials. However, typical sizes of quantum dots are in the region of several nanometers. As a consequence, active molecules will be a better choice when the gain shell in need is small and the density of optical gain is the same magnitude as in our case $[4,6,11,35,36]$.

In the end, we illustrate the difference between local and nonlocal cases for metal core-gain shell nanoparticles, as shown in Fig. 5. In this situation, the discrepancies are more than $5 \%$ in over $50 \%$ of the studied region, which indicates the importance of considering the nonlocality. In addition, we find a region in which the gain threshold for the nonlocal case is slightly lower than the local one [see the yellow circle region in Fig. 5(a)]. This unconventional phenomenon might result from the strong influence from the gold's interband transitions and the weak coupling between the optical modes and the thin gain medium.

For the refractive index of the background medium, we choose $n_{\mathrm{A}}=1$ only. It was reported that by increasing the refractive index of the background medium to an appropriate value lower gain fraction threshold as well as lower $\operatorname{Re}\left(n_{\mathrm{G}}\right)$ can be achieved in core-shell NPLs [7]. In this paper, our main aim is not to find a novel way to design a lower threshold NPLs but to pay attention to the nonlocal effects on the choice of physical parameters for designing these NPLs. For the compact Au core-gain shell nanoparticle lasers, the nonlocality (or spatial dispersion) must be taken into account to give the exact descriptions of the relevant parameters to realize the spaser. For instance, the prosed NPLs, which are composed of a gold core with $r_{\mathrm{c}}=7 \mathrm{~nm}$ and a dye-doped $\mathrm{SiO}_{2}$ active shell with $r_{\mathrm{s}}=10 \mathrm{~nm}$ and $n_{\mathrm{G}}=1.52$ embedded in the AlAs background dielectric with $\varepsilon_{\mathrm{A}}=3.25^{2}$, should have a modified $\lambda \sim 550 \mathrm{~nm}$ and a modified $G_{\text {th-non }} \sim 8.5 \times 10^{4} \mathrm{~cm}^{-1}$, instead of $\lambda \sim 570 \mathrm{~nm}$ and $G_{\text {th }} \sim 6.4 \times 10^{4} \mathrm{~cm}^{-1}$ for the local case. We should remark that, for an ultrasmall size $(2 \mathrm{~nm})$ as we adopt in our model, the quantum effects should be included, which needs a full quantum treatment. However, the semi- classical hydrodynamic model can capture the fundamental quantum-mechanical nature of the electrons [37] and give the qualitative agreement even when $r_{\mathrm{c}}=2 \mathrm{~nm}$ [38]. In addition, the nonlocality will, in general, result in the excitation of longitudinal modes, and these modes will become important above the plasma frequency [15], especially in the weak dissipative [26] or nondissipative [39] limit. However, due to the interband transition, the damping in high frequencies is extremely large, and hence these longitudinal peaks disappear in the extinction spectrum. As a result, the gain threshold is quite high in the high-frequency region.

\section{CONCLUSION}

In summary, we have suggested a nonlocal spaser containing a nonlocal nanosphere or nonlocal core-shell nanosphere and derived the spaser generation conditions for them. In general, the gain threshold and required refractive index for the nonlocal spaser are found to be larger than those for the local one. For an Au core-gain shell nanoparticle in the vacuum with a fixed outer radius, we obtain a broad region for $r_{\mathrm{c}}$, in which $G_{\text {th }}$ and $\operatorname{Re}\left(n_{\mathrm{G}}\right)$ keep almost unchanged. We expect that these results can be very important for prospective applications in the design of the ultrasmall nanoparticle spaser.

Here some comments are in order. First, for simplicity, we adopt a linearized version of the hydrodynamic Drude model and neglect the bound electron part of the permittivity, which may contribute to second-harmonic and third-harmonic generations [40,41]. Actually, if the gain medium is pumped strongly, the nonlinear effects may occur [40-42]. For further study on the spaser threshold gain with high- and short-time pump, the nonlinear version of the hydrodynamic Drude model with bound charge contribution should be adopted. In addition, a fully quantum-mechanical investigation using time-dependent density-functional theory is even better [43]. Moreover, many works on the calculation of spontaneous emission near spherical structures were proposed [44-46], and the change of spontaneous emission of radiation near a dielectric sphere could result in metal enhanced fluorescence or surface enhanced fluorescence. It is of interest to take into account the spontaneous emission within our model. Work along this line is in progress, and we shall report it elsewhere.

\section{ACKNOWLEDGMENTS}

This work was supported by the National Natural Science Foundation of China (Grants No. 11374223 and No. 11347105), the National Basic Research Program (Grant No. 2012CB921501), the Key Project in the Natural Science Foundation of the Jiangsu Education Committee (Grant No. 10 KJA140044), the Ph.D. Program Foundation of the Ministry of Education of China (Grant No. 20123201110010), the Natural Science Foundation for the Youth of Jiangsu Province (Grant No. BK20130284), and the Project Funded by the Priority Academic Program Development of Jiangsu Higher Education Institutions.
[1] D. J. Bergman and M. I. Stockman, Phys. Rev. Lett. 90, 027402 (2003).
[2] S. A. Maier, Plasmonics: Fundamentals and Applications (Springer, New York, 2007). 
[3] N. M. Lawandy, Appl. Phys. Lett. 90, 143104 (2007).

[4] P. Berini and I. De Leon, Nat. Photonics 6, 16 (2011).

[5] M. I. Stockman, Nat. Photonics 2, 327 (2008).

[6] M. A. Noginov, G. Zhu, A. M. Belgrave, R. Bakker, V. M. Shalaev, E. E. Narimanov, S. Stout, E. Herz, T. Suteewong, and U. Wiesner, Nature (London) 460, 1110 (2009).

[7] X. F. Li and S. F. Yu, Opt. Lett. 35, 2535 (2010).

[8] J. Pan, Z. Chen, J. Chen, P. Zhan, C. J. Tang, and Z. L. Wang, Opt. Lett. 37, 1181 (2012).

[9] N. Calander, D. Jin, and E. M. Goldys, J. Phys. Chem. C 116, 7546 (2012).

[10] S. Y. Liu, J. F. Li, F. Zhou, L. Gan, and Z. Y. Li, Opt. Lett. 36, 1296 (2011).

[11] X. Meng, A. V. Kildishev, K. Fujita, and V. M. Shalaev, Nano Lett. 13, 4106 (2013).

[12] X. Meng, U. Guler, A. V. Kildishev, K. Fujita, and V. M. Shalaev, Sci. Rep. 3, 1241 (2013).

[13] D. G. Baranov, A. P. Vinogradov, A. A. Lisyansky, Y. M. Strelniker, and D. J. Bergman, Opt. Lett. 38, 2002 (2013).

[14] D. B. Li and M. I. Stockman, Phys. Rev. Lett. 110, 106803 (2013).

[15] R. Ruppin, Phys. Rev. Lett. 31, 1434 (1973).

[16] C. Ciraci, R. T. Hill, J. J. Mock, Y. Urzhumov, A. I. FernandezDominguez, S. A. Maier, J. B. Pendry, A. Chilkoti, and D. R. Smith, Science 337, 1072 (2012).

[17] J. B. Pendry, A. Aubry, D. R. Smith, and S. A. Maier, Science 337, 549 (2012).

[18] A. Wiener, A. I. Fernandez-Dominguez, A. P. Horsfield, J. B. Pendry, and S. A. Maier, Nano Lett. 12, 3308 (2012).

[19] J. M. McMahon, S. K. Gray, and G. C. Schatz, Phys. Rev. Lett. 103, 097403 (2009).

[20] C. David and F. J. G. de Abajo, J. Phys. Chem. C 115, 19470 (2011).

[21] R. Esteban, A. G. Borisov, P. Nordlander, and J. Aizpurua, Nat. Commun. 3, 825 (2012).

[22] G. Toscano, S. Raza, S. Xiao, M. Wubs, A.-P. Jauho, S. I. Bozhevolnyi, and N. A. Mortensen, Opt. Lett. 37, 2538 (2012).

[23] G. Toscano, S. Raza, A. P. Jauho, N. A. Mortensen, and M. Wubs, Opt. Express 20, 4176 (2012).

[24] T. V. Teperik, P. Nordlander, J. Aizpurua, and A. G. Borisov, Phys. Rev. Lett. 110, 263901 (2013).
[25] Y. Luo, A. I. Fernandez-Dominguez, A. Wiener, S. A. Maier, and J. B. Pendry, Phys. Rev. Lett. 111, 093901 (2013).

[26] Y. Huang and L. Gao, J. Phys. Chem. C 117, 19203 (2013).

[27] E. S. Andrianov, A. A. Pukhov, A. V. Dorofeehko, A. P. Vinogradov, and A. A. Lisyansky, Opt. Express 19, 24849 (2011).

[28] G. S. Agarwal and S. V. ONeil, Phys. Rev. B 28, 487 (1983).

[29] V. V. Datsyuk, Ukr. J. Phys. 56, 122 (2011).

[30] R. D. Averitt, S. L. Westcott, and N. J. Halas, J Opt. Soc. Am. B 16, 1824 (1999).

[31] V. V. Datsyuk and O. M. Tovkach, J. Opt. Soc. Am. B 28, 1224 (2011).

[32] L. D. Landau, J. Phys. USSR 10, 25 (1946).

[33] N. M. Lawandy, Appl. Phys. Lett. 85, 5040 (2004).

[34] M. A. Noginov, G. Zhu, M. Bahoura, J. Adegoke, C. E. Small, B. A. Ritzo, V. P. Drachev, and V. M. Shalaev, Opt. Lett. 31, 3022 (2006).

[35] A. De Luca, M. Ferrie, S. Ravaine, M. La Deda, M. Infusino, A. R. Rashed, A. Veltri, A. Aradian, N. Scaramuzza, and G. Strangi, J. Mater. Chem. 22, 8846 (2012).

[36] K. G. Stamplecoskie, M. Grenier, and J. C. Scaiano, J. Am. Chem. Soc. 136, 2956 (2012).

[37] C. Ciracì, J. B. Pendry, and D. R. Smith, Chem. Phys. Chem. 14, 1109 (2013).

[38] L. Stella, P. Zhang, F. J. García-Vidal, A. Rubio, and P. GarcíaGonzález, J. Phys. Chem. C 117, 8941 (2013).

[39] M. I. Tribelsky, A. E. Miroshnichenko, and Y. S. Kivshar, Europhys. Lett. 97, 44005 (2012).

[40] C. Ciracì, E. Poutrina, M. Scalora, and D. R. Smith, Phys. Rev. B 86, 115451 (2012).

[41] M. Scalora, M. A. Vincenti, D. de Ceglia, V. Roppo, M. Centini, N. Akozbek, and M. J. Bloemer, Phys. Rev. A 82, 043828 (2010).

[42] J. E. Sipe, V. C. Y. So, M. Fukui, and G. I. Stegeman, Phys. Rev. B 21, 4389 (1980).

[43] D. C. Marinica, A. K. Kazansky, P. Nordlander, J. Aizpurua, and A. G. Borisov, Nano Lett. 12, 1333 (2012).

[44] V. V. Datsyuk, S. Juodkazis, and H. Misawa, J. Opt. Soc. Am. B 22, 1471 (2005).

[45] Y. Xu, R. K. Lee, and A. Yariv, Phys. Rev. A 61, 033807 (2000).

[46] H. T. Dung, L. Knöll, and D.-G. Welsch, Phys. Rev. A 62, 053804 (2000). 